\title{
Data acquisition schemes for continuous two-particle time-of-flight coincidence experiments
}

\author{
Andras Bodi \\ Paul Scherrer Institut, Villigen 5232, Switzerland \\ Bálint Sztáray \\ Eötvös Loránd University, Budapest 1117, Hungary and Department of Chemistry, University of North \\ Carolina, Chapel Hill, North Carolina 27599 \\ Tomas Baer \\ Department of Chemistry, University of North Carolina, Chapel Hill, North Carolina 27599 \\ Melanie Johnson and Thomas Gerber ${ }^{\text {a) }}$ \\ Paul Scherrer Institut, Villigen 5232, Switzerland
}

(Received 29 May 2007; accepted 4 August 2007; published online 23 August 2007)

\begin{abstract}
Three data acquisition schemes for two-particle coincidence experiments with a continuous source are discussed. The single-start/single-stop technique, implemented with a time-to-pulse-height converter, results in a complicated spectrum and breaks down severely at high count rates. The single-start/multiple-stop setup, based on a time-to-digital converter and the first choice in today's similar coincidence experiments, performs significantly better at high count rates, but its performance is still hampered if the time-of-flight range is large, and the false coincidence background is variable if the event frequency and the collection efficiency of the starts are both high. A straightforward, multistart/multistop setup is proposed for coincidence experiments. By collecting all detector data, it ensures the highest signal-to-noise ratio, constant background, and fast data acquisition and can now be easily constructed with commercially available time-to-digital converters. Analytical and numerically evaluated formulas are derived to characterize the performance of each setup in a variety of environments. Computer simulated spectra are presented to illustrate the analytically predicted features of the various raw time-of-flight distributions obtained with each technique. (C) 2007 American Institute of Physics. [DOI: 10.1063/1.2776012]
\end{abstract}

\section{INTRODUCTION}

There are three methods to carry out ion time-of-flight experiments: pulsed ion production, e.g., by a pulsed ionizing laser, ${ }^{1,2}$ pulsed ion extraction, ${ }^{3,4}$ and continuous ion extraction. ${ }^{5}$ If the ionization process is inherently continuous on the experimental time scale, such as a discharge lamp or a synchrotron light source, the first technique is not an option. If the aim is to select certain ions from the experimentally created multitude, the ion time-of-flight (TOF) analysis needs to be correlated with the selecting signal. A common experiment involves measuring ions in coincidence with energy selected photoelectrons, generally referred to as photoelectron photoion coincidence (PEPICO), in which ions of selected internal energies are of interest. ${ }^{5-7}$ Another selected photoion study involves doubly charged ions dissociating to two fragment ions, which can be investigated by detecting all fragment ions in mutual coincidence, a method termed photoion photoion coincidence (PIPICO). ${ }^{8}$ In some cases, the photoelectrons and both fragment ions are studied in triple coincidence (PEPIPICO). ${ }^{9}$

Due to the opposing requirement of high electron energy resolution, which in the past has dictated low extraction

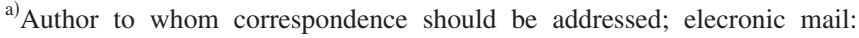
thomas.gerber@psi.ch
}

fields, and the desired high ion collection efficiency, which is enhanced by high extraction fields, coincidence experiments often pulse the ions out of the ionization region once the photoelectron has been detected. ${ }^{10-15}$ Pulsed extraction means that ions are accumulated in the ionization region for a certain time until they are extracted following a start pulse. This may lead to more false coincidences and possibly many more counts per TOF cycle than in a continuous experiment. These issues have been addressed by Luhmann. ${ }^{16}$ Powis and Downie have proposed a random start generator to account for the false coincidences in pulsed techniques experimentally. ${ }^{17}$

In this article, we deal exclusively with the case of a continuous extraction field, for which there are two common data acquisition setups. Time-to-pulse-height converters (TPHCs) also known as time-to-amplitude converters (TACs) start the TOF analysis when a start signal arrives, count until the first stop signal is obtained, and supply a pulse, the height of which is a function of the elapsed time of flight. ${ }^{5}$ This results in single-start/single-stop (SS) data acquisition. Start signals are lost while the TPHC is waiting for the stop to arrive, and further stop signals are also lost when the first stop signal (either the desired stop or a false coincidence) is recorded, i.e., the TPHC may be paralyzed as it is unable to collect multiple stops in a TOF cycle. Time-to- 
digital converters (TDC's) are used to circumvent the latter shortcoming of the TPHC's, namely, the inability to collect more than one stop for a single start signal. TDC's wait for a start, count for a preset time interval, and record all stops that arrive with a given time resolution. ${ }^{18}$ However, start signals are still lost while the TDC acquisition cycle is running; hence it is a single-start/multiple-stop (SM) setup.

When the ion production and extraction are both continuous, the general agreement appears to be that the background, false coincidence stop signal is uncorrelated with the TOF save for paralysis effects, thus the SM background should be constant in the whole TOF range. ${ }^{16,17,19,20}$ However, we will show that this is only exactly true when all experimental data are registered in the multiple-start/ multiple-stop (MM) scheme. This can be achieved with a master clock to time the start and stop events and by establishing the correlation between the two spike trains. Due to the fact that ions born in different stages of an SS or SM experiment (a TOF cycle running versus the idle time periods in between) have different chances of contributing to the measured false coincidence signal, the false coincidence signal is not uncorrelated with the start signal. As supported by simulations, we will show that this correlation may have a detrimental effect on the quality of the TOF spectra when both the ionization frequency and the start collection efficiency are high.

It is the expected high ionization rate at the continuous, newly built imaging photoelectron photoion coincidence experiment (iPEPICO) at the X04DB vacuum ultraviolet (VUV) beamline of the Swiss Light Source (Villigen, Switzerland) that has prompted us to propose a new way to process the detector signals and to reexamine the issue of false coincidences and the resulting uncertainties in the TOF spectra. In this experiment, both electrons and ions are extracted by a continuous field of between 20 and $40 \mathrm{~V} / \mathrm{cm}$. Electron energy resolution of less than $10 \mathrm{meV}$ has already been obtained in a laboratory based experiment, ${ }^{5,6}$ a resolution that is expected to improve to $1 \mathrm{meV}$ with the new synchrotron based experiment.

\section{GENERAL PRINCIPLES}

The experiment is defined by the ionization event frequency $(f)$, the collection efficiencies for start and stop signals, i.e., electrons and ions in an electron-ion coincidence setup $\left(\eta_{e}, \eta_{i}\right)$, and the probability distribution of the ion TOF $\left[p(t), \int_{0}^{t_{\max }} p(t) d t=1\right]$. The information in the PEPICO TOF distribution is threefold: the peak positions correspond to ion masses, the relative peak areas correspond to ion abundances, and the peak shapes can be used to derive either direct kinetic information of the photodissociation ${ }^{5,21}$ or a measure of the kinetic energy release in its course. ${ }^{22} \mathrm{We}$ assume a $p(t)$ with two arbitrarily shaped peaks, both of which have a width $w$ and 0 intensity outside the peaks. The peaks occupy the $\left[t_{A}, t_{A}+w\right]$ and $\left[t_{B}, t_{B}+w\right]$ time-of-flight intervals. Let $r \equiv \int_{t_{A}}^{t_{A}+w} p(t) d t / \int_{0}^{t_{\max }} p(t) d t=\int_{t_{A}}^{t_{A}+w} p(t) d t=c_{A} / c$, where $c=c_{A}+c_{B}$, i.e., the total number of true coincidences and $c_{A}$ and $c_{B}$ denote the number of true coincidences observed for peaks $A$ and $B$, respectively. True coincidences are the ones for which the start and the stop signals stem from a single ionization event. All others are false coincidences, but only start and stop signals from different ionization events are considered here. Surface electrons and background ions are examples of other potential sources of false coincidences. The experiment runs for $T_{\text {tot }}$ time, during which $c_{A}$ and $c_{B}$ true coincidences are collected in the two TOF peaks. We choose the statistical uncertainty in the peak area ratio $\delta r$ as a quality indicator, since peak shapes are also closely related to relative peak areas but are more difficult to describe generally. The statistical uncertainty in $c_{A}$ and $c_{B}\left(\delta c_{A}\right.$ and $\left.\delta c_{B}\right)$ as well as in the false coincidence background $c_{F A}$ and $c_{F B}$ $\left(\delta c_{F A}\right.$ and $\left.\delta c_{F B}\right)$ contribute to the uncertainty in the peak area ratio $r$. The variables $c_{A}, c_{B}, c_{F A}$, and $c_{F B}$ are uncorrelated (hence the uncertainty in the total peak area is $\left.\delta \bar{c}_{X}^{2}=\delta c_{X}^{2}+\delta c_{F X}^{2}, X=A, B\right)$, and, according to the law of error propagation ${ }^{23} \delta r^{2}=\Sigma_{X}\left(\partial r / \partial c_{X}\right)^{2} \delta \bar{c}_{X}^{2}$ the uncertainty in the peak area ratio will be

$$
\delta r=\frac{\sqrt{c_{A}^{2}\left(\delta c_{B}^{2}+\delta c_{F B}^{2}\right)+c_{B}^{2}\left(\delta c_{A}^{2}+\delta c_{F A}^{2}\right)}}{\left(c_{A}+c_{B}\right)^{2}} .
$$

Furthermore, let us assume the Poisson standard deviation of $\delta c_{X}=\sqrt{c_{X}}$.

In an ideal experiment with an ionization event frequency $f$ and in which false coincidences are absent, $c_{A}=r c$ and $c_{B}=(1-r) c$, where $c=\eta_{e} \eta_{i} f T_{\text {tot }}$ is the total number of registered true coincidences,

$$
\delta r=\sqrt{\frac{r(1-r)}{c}} \propto \frac{1}{\sqrt{T_{\text {tot }}}} .
$$

Consequently, the uncertainty of the peak area ratio is inversely proportional to the square root of the measurement time. This relation will transpire to be the limiting behavior for all (SS, SM, and MM) data acquisition techniques.

Events are normally collected in digital time bins, and the average number of random counts in a start or stop time bin is governed by the Poisson distribution,

$$
P_{k}=\frac{\bar{N}^{k}}{k !} e^{-\bar{N}},
$$

where $k=0,1,2, \ldots$, is the number of counts expected in a time bin and $\bar{N}=f t_{\text {time bin }}$ is the expectation value of the number of counts in a time bin.

Luhmann has extensively studied the paralytic effect of more than one signal coinciding in a single channel. ${ }^{16}$ In the pulsed extraction mode, such events may be a problem. However, in the continuous extraction case considered here, if $P_{1}<0.01$, multiple counts become negligible relative to single hits $(<0.5 \%)$, and with an attainable time resolution of $10 \mathrm{~ns}$, this translates into a maximal detection frequency of $1 \mathrm{MHz}$. If individual events are counted, electron and ion detectors are rarely used above this rate; therefore, we choose to neglect the Poisson paralysis in the derivation.

\section{STATISTICAL UNCERTAINTY IN THE TOF SPECTRA}

The description of the TOF distributions and their statistical handling is simplest for the MM case and most demand- 
ing for the SS case, in reverse order of the complexity of their implementation. Therefore, the MM TOF distributions are described first. The MATHEMATICA 4.1 software package was used in the derivation and evaluation of the SS, SM, and MM formulas. ${ }^{24}$

Multiple-start/multiple-stop data acquisition. All start signals are correlated with all stop signals within the relevant time interval. Therefore if exactly one true stop belongs to each start signal, the total number of true coincidences will be

$$
c_{\mathrm{MM}}=\eta_{e} \eta_{i} f T_{\text {tot }} \quad c_{A, \mathrm{MM}}=r c_{\mathrm{MM}}, \quad c_{B, \mathrm{MM}}=(1-r) c_{\mathrm{MM}} .
$$

Every stop signal has the same chance of contributing to the false coincidence background in the TOF spectrum according to Eq. (3). Thus, the false coincidence background will be uniform along the spectrum, and its total intensity along peak width $w$ is given as a product of the total number of starts $\left(\eta_{e} f T_{\text {tot }}\right)$ and the average number of ion counts $\left(\eta_{i} f w\right)$ in time interval $w$,

$$
c_{F A, \mathrm{MM}}=c_{F B, \mathrm{MM}}=\eta_{e} \eta_{i} f^{2} T_{\mathrm{tot}} w .
$$

Thus, the statistical uncertainties are according to the Poisson standard deviation,

$$
\begin{aligned}
& \delta c_{A, \mathrm{MM}}^{2}+\delta c_{F A, \mathrm{MM}}^{2}=\eta_{e} \eta_{i} f T_{\text {tot }}(r+f w), \\
& \delta c_{B, \mathrm{MM}}^{2}+\delta c_{F B, \mathrm{MM}}^{2}=\eta_{e} \eta_{i} f T_{\text {tot }}(1-r+f w) .
\end{aligned}
$$

Substituting Eqs. (5) and (6) into Eq. (1) the uncertainty in the peak area ratio becomes

$$
\delta r_{\mathrm{MM}}=\sqrt{\frac{f w+r(1-r)(1-2 f w)}{\eta_{e} \eta_{i} f T_{\text {tot }}}} .
$$

$=\frac{1}{2} \sqrt{(1+2 f w) / \eta_{e} \eta_{i} f T_{\text {tot }}}$.

Single-start/multiple-stop data acquisition. In this setup, not all start signals are recorded, because the start input is dead during the TOF cycles. This setup closely resembles the one described by Gellender and Baker, ${ }^{19}$ with the important difference that they suppose a homogeneous false coincidence background, which is well founded in the $\eta_{e} \approx 10^{-5}$ range but, as will be shown later, is inaccurate when the event frequency and the electron collection efficiency are both high. The total number of recorded starts equals the total measurement time $\left(T_{\text {tot }}\right)$ divided by the length of each cycle $\left(t_{\max }\right)$ plus the average time $\left(1 / f \eta_{e}\right)$ the TDC waits for the next start signal after the completion of the previous cycle.

$$
N_{\mathrm{SM}}=\frac{T_{\mathrm{tot}}}{t_{\max }+1 / f \eta_{e}}
$$

The true coincidence counts are, thus,

$$
\begin{aligned}
& c_{\mathrm{SM}}=N_{\mathrm{SM}} \eta_{i}=\frac{T_{\mathrm{tot}} \eta_{i}}{t_{\max }+1 / f \eta_{e}}, \\
& c_{A, \mathrm{SM}}=r c_{\mathrm{SM}}, \quad c_{B, \mathrm{SM}}=(1-r) c_{\mathrm{SM}} .
\end{aligned}
$$

Three time lines can be distinguished for false coincidence signals: (a) Stop signals from ionization events having taken place before the current cycle started while the TDC was waiting for stops in a previous active cycle. Because the start input of the TDC is dead during the previous TOF cycle, the start signal is lost with certainty, and the contribution to the false coincidence background is $f \eta_{i}$.

(b) Stop signals from ionization events having taken place before the current cycle started while the TDC was inactive. Because the start input of the TDC was active, the probability that the start signal was lost is only $\left(1-\eta_{e}\right)$, which results in a $\left(1-\eta_{e}\right) f \eta_{i}$ contribution to the false coincidence background.

(c) Stop signals from ionization events taking place after the current cycle started. As in (a), the start signal is lost with certainty, since the start input of the TDC is dead in the current cycle. This results in an $f \eta_{i}$ contribution to the false coincidence background.

The stop signal is partitioned between cases (a) and (b) and (c), that is, the event resulting in the false stop taking place before the current start versus that after current start, according to the normalized TOF distribution $p(t)$. At time of flight $t_{0}$, the ratio can be calculated as $\int_{t_{0}}^{t_{\max }} p(t) d t: \int_{0}^{t_{0}} p(t) d t$. Cases (a) and (b) still need to be separated. Let $\beta(t)$ denote the probability that the TDC was listening for a start at time $t$ preceding the current start. $\beta(0)=1$, since the TDC was certainly listening for starts when it captured the current start, and start signals come with an $f \eta_{e}$ frequency as a Poisson series, hence the probability distribution of the gap lengths between the TOF cycles can be described by $\beta(t)=e^{-f \eta_{e} t}$. The ratios (a) : (b) : (c) are consequently $\int_{t_{0}}^{t_{\max }}$ $\left[1-\beta\left(t-t_{0}\right)\right] p(t) d t: \int_{t_{0}}^{t_{\max }} \beta\left(t-t_{0}\right) p(t) d t: \int_{0}^{t_{0}} p(t) d t$. The total false coincidence frequency will be the sum of the three components,

$$
\begin{aligned}
f_{F, \mathrm{SM}}\left(t_{0}\right)= & f \eta_{i}\left\{\int_{t_{0}}^{t_{\max }}\left[1-\beta\left(t-t_{0}\right)\right] p(t) d t\right. \\
& \left.+\left(1-\eta_{e}\right) \int_{t_{0}}^{t_{\max }} \beta\left(t-t_{0}\right) p(t) d t+\int_{0}^{t_{0}} p(t) d t\right\} \\
= & f \eta_{i}\left[1-\int_{t_{0}}^{t_{\max }} p(t) \eta_{e} e^{-f \eta_{e}\left(t-t_{0}\right)} d t\right] .
\end{aligned}
$$

The false coincidence counts for peaks $A$ and $B$ are obtained by the integrals

$$
\begin{aligned}
c_{F A, \mathrm{SM}} & =N_{\mathrm{SM}} \int_{t_{A}}^{t_{A}+w} f_{F, \mathrm{SM}}\left(t_{0}\right) d t_{0}, \\
c_{F B, \mathrm{SM}} & =N_{\mathrm{SM}} \int_{t_{B}}^{t_{B}+w} f_{F, \mathrm{SM}}\left(t_{0}\right) d t_{0} .
\end{aligned}
$$

The error analysis can then be carried out according to Eq. (1).

Two crucial qualitative differences emerge when comparing the SM and MM TOF distributions. First, the false coincidence background is not uniform in the SM spectra, and its variation depends on the ionization frequency and the start collection efficiency. The changing false coincidence 
background of the SM spectrum may affect the peak shapes and shift the observed peak centroids. Second, the SM uncertainty depends on the maximum flight time $t_{\max }$ predominantly through the number of cycles that fit in the total measurement time [Eq. (8)], whereas the MM uncertainty does not. This means that it is not possible to extend the SM TOF range by, for instance, a reflectron, without paying a penalty in the total accumulated signal and the signal-to-noise ratio. No such limitation pertains to the MM case.

As the analytical form of the SM uncertainty is complicated even for the simplest of TOF distributions and also lacks guidance as to which parameters are of importance, one can take an indirect approach. The ratio of the uncertainties in the SM and MM spectra yields a relatively simple formula in the $w \rightarrow 0$ limit, i.e., if the inhomogeneity in the false coincidence background [the integral in Eq. (10)] can be neglected. The SM spectrum thus becomes an MM spectrum with fewer starts and proportionally fewer true and false coincidences, and the $w \rightarrow 0$ limit can be used together with the MM uncertainty expression in Eq. (7) to derive $\delta r_{\mathrm{SM}}$ for $r=50 \%$,

$$
\lim _{w \rightarrow 0} \frac{\delta r_{\mathrm{SM}}}{\delta r_{\mathrm{MM}}}=\sqrt{\frac{c_{\mathrm{MM}}}{c_{\mathrm{SM}}}}=\sqrt{1+\eta_{e} f t_{\max }},
$$

and, by multiplying by $\delta r_{\mathrm{MM}}$,

$$
\delta r_{\mathrm{SM}} \approx \frac{1}{2} \sqrt{\frac{\left(1+\eta_{e} f t_{\max }\right)(1+2 f w)}{\eta_{e} \eta_{i} f T_{\mathrm{tot}}}} .
$$

Based on Eq. (12), it is evident that the advantage of the MM setup is largest when the electron collection efficiency, the event frequency and the time-of-flight range are large. At the same time, the performance ratio of the SM and MM setups is independent of the stop collection efficiency, and the MM and SM setups converge if the number of missed starts during a TOF cycle $\left(\eta_{e} f t_{\text {max }}\right)$ decreases. The SM uncertainty function shows a minimum at $f \approx 1 / \sqrt{2 \eta_{e} t_{\max } w}$. Gellender and Baker arrive at an equivalent formula in their work, Eq. (7) in Ref. 19. They report this frequency as the exact maximum of the signal-to-noise ratio, whereas it is only an approximation neglecting the correlation of the false coincidence background with the recorded start signals. Indeed, the correlation is negligible in the start collection efficiency regime they studied $\left(\eta_{e}=10^{-5}\right)$.

Single-start/single-stop data acquisition. In comparison with the SM setup, the time-of-flight counting stops when the first stop signal is received in this scheme. Therefore, the resulting time-of-flight distribution is essentially a modified SM spectrum in which each value is multiplied by the probability that no stops have been registered since the start signal. True coincidences, however, do not decrease the probability of registering another true coincidence, provided that only one true stop belongs to each start. On the other hand, false coincidence levels are decreased by both true and false coincidence counts registered at earlier flight times. In contrast with the SM setup, the TOF clock is restarted every time a stop signal is recorded. Therefore, the total number of recorded starts will be higher during a given measurement time.
The average number of recorded true coincidences per TOF cycle in peaks $A$ and $B$ will thus be

$\chi_{A, \mathrm{SS}}=\eta_{i} \int_{t_{A}}^{t_{A}+w} p(t) \exp \left[-\int_{0}^{t} f_{F, \mathrm{SM}}\left(t_{0}\right) d t_{0}\right] d t=\eta_{i} r / \sigma_{A}$,
$\chi_{B, \mathrm{SS}}=\eta_{i} \int_{t_{B}}^{t_{B}+w} p(t) \exp \left[-\int_{0}^{t} f_{F, \mathrm{SM}}\left(t_{0}\right) d t_{0}\right] d t=\eta_{i}(1-r) / \sigma_{B}$,

where the exponential part stands for the probability that the detector records a false coincidence before recording the true stop in the time range of the respective peaks [cf. Eq. (10)], and $\sigma_{X}(X=A, B)$ describes the paralysis of each peak. The false coincidence signal is also paralyzed by the true coincidences, thus the false coincidence background of the TOF peaks involves a slightly different integral,

$$
\begin{aligned}
\chi_{F A, \mathrm{SS}} & =\int_{t_{A}}^{t_{A}+w} f_{F, \mathrm{SM}}\left(t_{1}\right) \exp \left[-\int_{0}^{t_{1}} f_{F, \mathrm{SM}}\left(t_{0}\right)+\eta_{i} p\left(t_{0}\right) d t_{0}\right] d t_{1} \\
& =\int_{t_{A}}^{t_{A}+w} f_{F, \mathrm{SM}}\left(t_{1}\right) \psi\left(t_{1}\right) d t_{1} \\
\chi_{F B, \mathrm{SS}} & =\int_{t_{B}}^{t_{B}+w} f_{F, \mathrm{SM}}\left(t_{1}\right) \psi\left(t_{1}\right) d t_{1}
\end{aligned}
$$

where $\psi\left(t_{1}\right)$ is the probability that neither a true nor a false stop signal is detected until $t_{1}$ in a TOF cycle.

The number of TOF cycles is calculated by taking into account how much time it takes on average for the TOF cycle to finish either by detecting a stop or by exceeding the $t_{\max }$ limit without a stop, in addition to the time the TPHC waits for the start signals as in Eq. (8).

$$
N_{\mathrm{SS}}=\frac{T_{\mathrm{tot}}}{1 / f \eta_{e}+\int_{0}^{t_{\max }} \psi\left(t_{1}\right) d t_{1}},
$$

where $\int_{0}^{t_{\max }} \psi\left(t_{1}\right) d t_{1}$ yields the average time it takes for the TOF cycle to complete.

The true and false coincidence counts can be obtained based on the number of cycles and the average values of the counts per cycle: $c_{X, \mathrm{SS}}=\chi_{X} N_{\mathrm{SS}}$. The true coincidence ratio is $r_{\mathrm{SS}}=c_{A, \mathrm{SS}} \sigma_{A} /\left(c_{A, \mathrm{SS}} \sigma_{A}+c_{B, \mathrm{SS}} \sigma_{B}\right)$ for the SS spectrum. Therefore [cf. Eq. (1)],

$\delta r_{\mathrm{SS}}=\frac{\sigma_{A} \sigma_{B} \sqrt{c_{A, \mathrm{SS}}^{2}\left(\delta c_{B, \mathrm{SS}}^{2}+\delta c_{F B, \mathrm{SS}}^{2}\right)+c_{B, \mathrm{SS}}^{2}\left(\delta c_{A, \mathrm{SS}}^{2}+\delta c_{F A, \mathrm{SS}}^{2}\right)}}{\left(c_{A, \mathrm{SS}} \sigma_{A}+c_{B, \mathrm{SS}} \sigma_{B}\right)^{2}}$.

Equations (13)-(16) do not have an analytical form even for the simplest TOF distributions; they have to be evaluated numerically instead. However, two approximations can be employed. First, in the limit of low start collection efficiencies, the false coincidence ion signal can be approximated as constant $f \eta_{i}$, neglecting the integral in Eq. (10) in the same way as in obtaining Eq. (12). Second, when signal levels are high and paralysis plays a major role, the paralysis of the false coincidence signal due to true coincidences can be neglected compared to the paralysis due to false coincidences. 


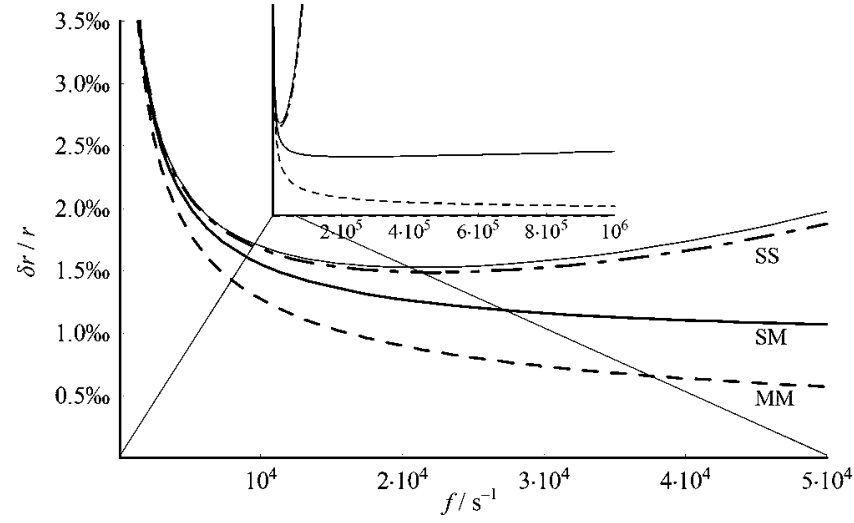

FIG. 1. The effect of the event frequency on the relative peak area uncertainty in the acquired TOF distribution. The modeled spectrum consists of two equal, rectangular peaks in the $[0, w]$ and $\left[t_{\max }-w, t_{\max }\right]$ time range. Acquisition parameters: $t_{\max }=200 \mu \mathrm{s}, w=200 \mathrm{~ns}, T_{\text {tot }}=1000 \mathrm{~s}, \eta_{i}=25 \%$, and $\eta_{e}=25 \%$. The insert shows the uncertainty behavior over a larger frequency interval. The thin curve running parallel to the SS curve is the one evaluated with the approximate Eq. (17) formula.

Supposing the peaks are rectangular (of constant height) in the $[0, w],\left[t_{\max }-w, t_{\max }\right]$ TOF ranges for peaks $A$ and $B$, respectively, the SS statistical uncertainty can be evaluated in the $w \rightarrow 0$ limit.

$$
\lim _{w \rightarrow 0} \frac{\delta r_{\mathrm{SS}}}{\delta r_{\mathrm{MM}}} \approx \sqrt{\frac{(1+q)\left[\eta_{e}(q-1)+p q\right]}{2 p q}},
$$

where $p=\eta_{i} e^{\eta_{i} / 2}$ and $q=e^{\eta_{i} / f t_{\max }}$. Thus,

$$
\delta r_{\mathrm{SS}} \approx \frac{1}{2} \sqrt{\frac{(1+q)\left[\eta_{e}(q-1)+p q\right](1+2 f w)}{2 p q \eta_{e} \eta_{i} f T_{\mathrm{tot}}}} .
$$

Equations (7), (12), and (17) enable a simple, analytic comparison of the statistical uncertainty of the peak areas for the three setups. The integral formalism can be employed to derive an iterative false coincidence correction scheme for the SM and SS setups [Eqs. (9)-(11) and (13)-(16), respectively], which makes it possible to correct experimental data accurately. The first guess for $p(t)$ is typically straightforward, and computer simulations have shown that $p(t)$ converges generally quickly to an apparently well-defined minimum.

Performance comparison. The performance of the SS, $\mathrm{SM}$, and MM data acquisition schemes is compared in Figs. 1-3. The parameters were chosen to be in a range typically encountered in two-particle time-of-flight coincidence experiments. The peak area is distributed evenly $(r=50 \%)$ and rectangular peaks are assumed with $p(t)=1 /(2 w)$ for $0<t<w$ and for $t_{B}<t<t_{B}+w=t_{\max }$, whereas $p(t)=0$ otherwise. Figure 1 illustrates the uncertainty in the peak area ratios as a function of the event frequency. There are two SS curves with the thin one being the approximate result from Eq. (17), and the thicker one the exact uncertainty with the SS integrals numerically evaluated. From here on, the exact results are plotted, but the approximate formulas (12) and (17) perform fairly well. At low frequencies, the SS uncertainty is large, because few events are recorded. As the frequency increases, the uncertainty decreases. However, as the frequency becomes even larger, the paralysis due to false

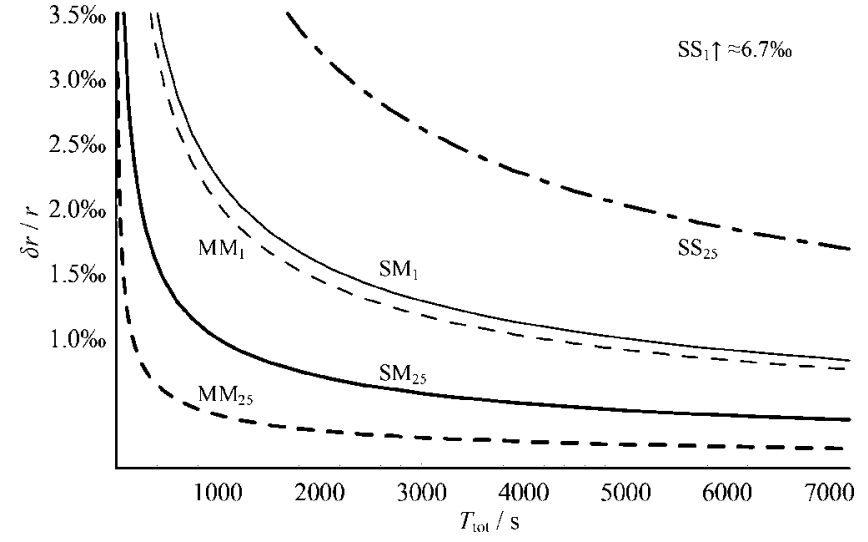

FIG. 2. The relative uncertainty of the peak area as a function of $T_{\text {tot }}$ for the two indicated start collection efficiencies; $1 \%$ and $25 \%$. The assumed twopeak spectrum is the same as that in Fig. 1. Other acquisition parameters were $f=10^{5} \mathrm{~s}^{-1}, t_{\max }=200 \mu \mathrm{s}, w=200 \mathrm{~ns}$, and $\eta_{i}=25 \%$.

coincidences starts to affect the uncertainty relatively soon, and the SS setup becomes progressively worse relative to the $\mathrm{SM}$ result above $10^{5} \mathrm{~s}^{-1}$ event frequency. Thus, the SS uncertainty has a well-defined minimum at a relatively low ionization frequency. Theoretically, the SS spectrum may actually be better than the SM spectrum, especially at high collection efficiencies and TOF ranges, where $N_{\mathrm{SS}}$ may be significantly higher than $N_{\mathrm{SM}}$. Still, the SS and SM results are significantly inferior to the MM result over the whole frequency range.

Figure 2 illustrates the $T_{\text {tot }}$ dependence of the uncertainty with two different start collection efficiencies: $1 \%$ and $25 \%$. The SS setup is clearly inferior to the SM and MM cases, with the $\mathrm{SS}_{1}$ curve being outside the plotted range. It is, however, interesting that the true advantage of the MM setup is only conspicuous at high start collection efficiencies [see Eq. (12)]. The total time-of-flight range also affects the SS and SM spectra, as seen in Fig. 3. Although an increased total TOF has an attenuated effect on the SM spectrum, the relative uncertainty on the SM TOF distribution is more than twice that of the MM one at a $t_{\max }$ higher than $100 \mu \mathrm{s}$.

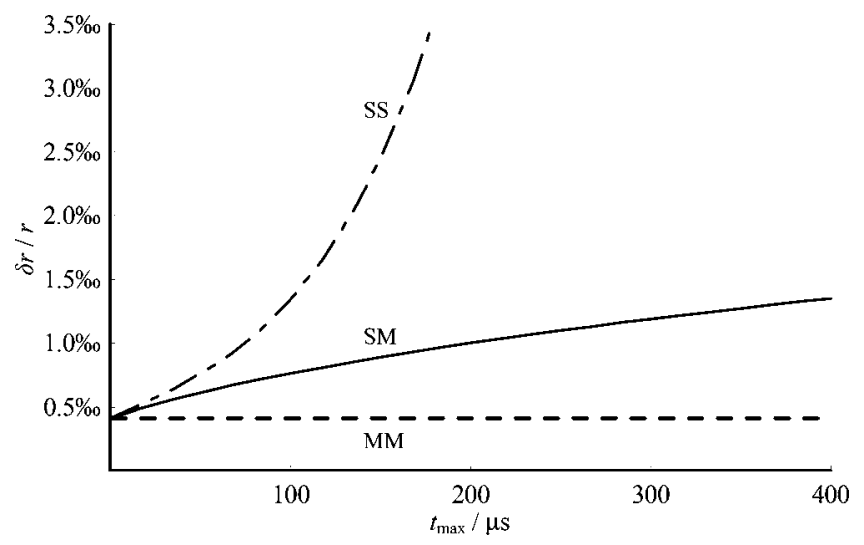

FIG. 3. The relative uncertainty of the peak area as a function of $t_{\max }$ for the following acquisition parameters: $f=10^{5} \mathrm{~s}^{-1}, w=200 \mathrm{~ns}, T_{\mathrm{tot}}=1000 \mathrm{~s}$, $\eta_{i}=25 \%$, and $\eta_{e}=25 \%$. The assumed two-peak spectrum is the same as that in Fig. 1. 


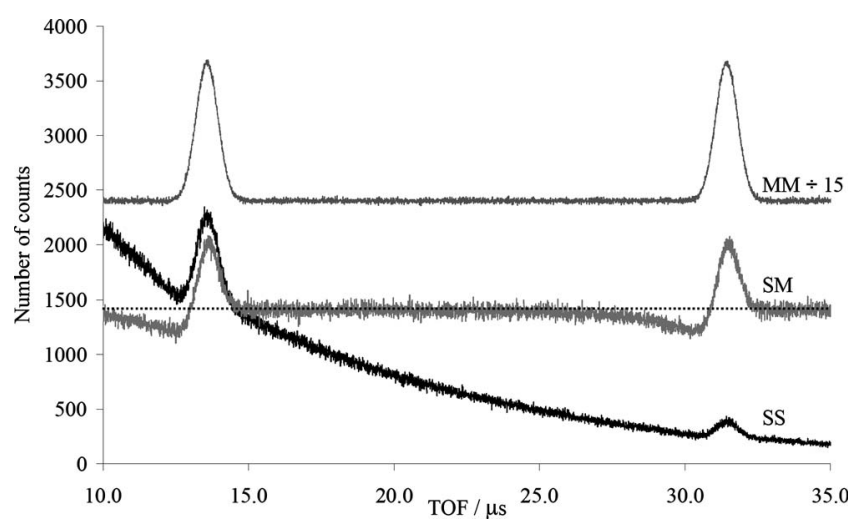

FIG. 4. TOF spectra with two Gaussian peaks obtained with SS, SM, and MM techniques. Acquisition parameters: $f=10^{6} \mathrm{~s}^{-1}, T_{\mathrm{tot}}=120 \mathrm{~s}, \eta_{i}=10 \%$, $\eta_{e}=50 \%$, and $t_{\max }=50 \mu \mathrm{s}$.

\section{SIMULATIONS}

Computer simulations have also been carried out to check the derived noise-correction schemes iteratively and to illustrate the predicted features in the false-coincidence background. A simple spectrum is presented with two Gaussian peaks at 13.5 and $31.5 \mu$ s in Figs. 4 and 5. A Poisson event series was generated, from which $50 \%$ of the start signals and $90 \%$ of the stop signals were discarded. The raw simulated spectra with the SS, SM, and MM data acquisition techniques are shown in Fig. 4, with the MM signal divided by 15 to bring the three on the same scale. A most interesting feature is how the SM false-coincidence background changes before and under the peak, an effect emphasized by the horizontal dotted line to guide the eye, and one that actually distorts the peak shapes in the spectrum. The SS spectrum is also affected, but the effect is small compared to the paralysis of the acquisition electronics. Even though the false coincidence barely remains a function of the SM and SS TOF's as the start collection efficiency decreases to $1 \%-10 \%, 50 \%$ collection efficiencies are not unheard of, ${ }^{25}$ and the nonlinear distortion of the spectrum is clearly an undesired consequence of these data acquisition techniques. We have used the derived formulas to iteratively refine the SS and SM spectra, and the results (together with the MM result with the false-coincidence background subtracted) are shown in Fig. 5. The errors in the observed peak area ratio $(33.8 \%$, $3.3 \%$, and $2.2 \%$ for the SS, SM, and MM setups, respectively) agree with the spectral quality observed by the bare eye. The dips in the false coincidence background are efficiently accounted for with the known electron collection efficiency for both the SM and SS setups, and it is also interesting to see how the noise level in the SS setup increases with the flight time due to the paralysis of the detection electronics.

\section{DISCUSSION}

Three data acquisition techniques have been investigated for continuous-source two-particle time-of-flight coincidence experiments. The false coincidence background has been described for the various data acquisition techniques, and

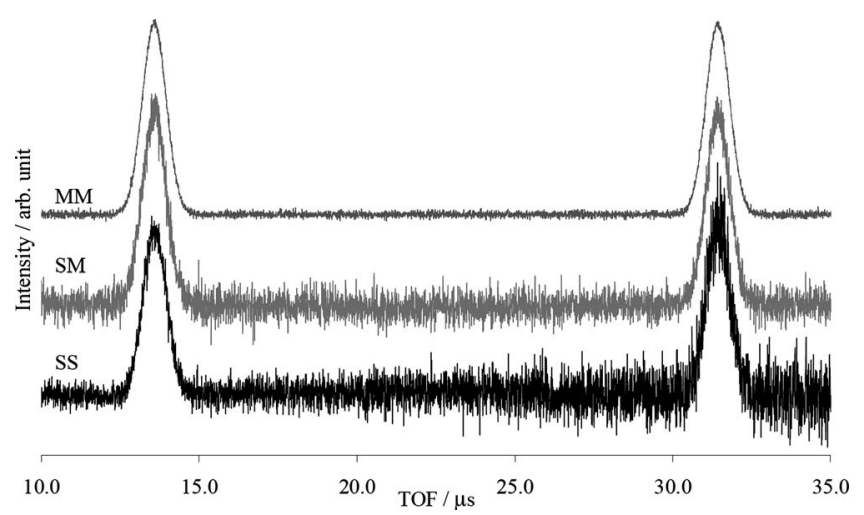

FIG. 5. Corrected SS, SM, and MM spectra based on the raw spectra in Fig. 4. The three spectra have been normalized to the same height with their base lines offset for clarity. The errors in the observed peak area ratios are $33.8 \%, 3.3 \%$, and $2.2 \%$ for the three setups, respectively.

together with the true coincidence signal, it is used to calculate the statistical uncertainty of the peak ratios in the TOF distribution. The conventional single-start/single-stop technique breaks down at high event frequencies and is unsuitable for high intensity experiments. It is also shown that the false-coincidence background can exhibit significant variations in the single-start/multiple-stop setup, especially at high start collection efficiencies. The advantage of collecting all detector data in the multiple-start/multiple-stop setup is largest when the start collection efficiency and the total TOF range are high. Nevertheless, the performance gain of the MM setup is significant across a broad range of parameters. Furthermore, the MM setup is not affected by long flight times, making it easy to improve, eg., the mass resolution by increasing the TOF range without compromising the total measurement time.

\section{ACKNOWLEDGMENTS}

The authors are thankful to Swiss Federal Office of Energy (BFE) and to the U.S. Department of Energy. The cooperation between the U.S. and Hungarian groups was supported by a joint Hungarian MTA-OTKA and U.S. National Science Foundation Grant. The authors are grateful to the Országos Tudományos Kutatási Alap (Grant Nos. F61153 and T60679). The comments and suggestions from Dr. Nicholas S. Shuman are also thankfully acknowledged.

\footnotetext{
${ }^{1}$ F. Merkt, Annu. Rev. Phys. Chem. 48, 675 (1997).

${ }^{2}$ R. A. Dressler et al., J. Chem. Phys. 125, 132306 (2006).

${ }^{3}$ M. Richard-Viard, A. Delboulbe, and M. Vervloet, Chem. Phys. 209, 159 (1996).

${ }^{4}$ X. M. Qian et al., Rev. Sci. Instrum. 74, 4096 (2003).

${ }^{5}$ T. Baer, B. Sztáray, J. P. Kercher, A. F. Lago, A. Bodi, C. Skull, and D. Palathinkal, Phys. Chem. Chem. Phys. 7, 1507 (2005).

${ }^{6}$ B. Sztáray and T. Baer, Rev. Sci. Instrum. 74, 3763 (2003).

${ }^{7}$ G. K. Jarvis, K. J. Boyle, C. A. Mayhew, and R. P. Tuckett, J. Phys. Chem. A 102, 3230 (1998).

${ }^{8}$ T. Ibuki et al., Chem. Phys. 314, 119 (2005).

${ }^{9}$ R. D. Molloy and J. H. D. Eland, Chem. Phys. Lett. 421, 31 (2006).

${ }^{10}$ K. Norwood and C. Y. Ng, J. Chem. Phys. 91, 2898 (1989).

${ }^{11}$ S. Y. Chiang and C. I. Ma, J. Phys. Chem. A 104, 1991 (2000).

${ }^{12}$ O. Dutuit, T. Baer, C. Metayer, and J. Lemaire, Int. J. Mass Spectrom. Ion Process. 110, 67 (1991).

${ }^{13}$ J. Mähnert, H. Baumgärtel, and K. M. Weitzel, J. Phys. Chem. 107, 6667 (1997)
} 
${ }^{14}$ T. Kinugawa, A. M. Hodgekins, and J. H. D. Eland, Chem. Phys. Lett. 368, 276 (2003).

${ }^{15}$ R. Thissen, C. Alcaraz, J. W. Hepburn, M. Vervloet, and O. Dutuit, Int. J. Mass. Spectrom. 199, 201 (2000).

${ }^{16}$ T. Luhmann, Rev. Sci. Instrum. 68, 2347 (1997).

${ }^{17}$ I. Powis and P. Downie, Rev. Sci. Instrum. 69, 3142 (1998).

${ }^{18}$ M. Lebech, J. C. Houver, and D. Dowek, Rev. Sci. Instrum. 73, 1866 (2002).

${ }^{19}$ M. E. Gellender and A. D. Baker, Int. J. Mass Spectrom. Ion Phys. 17, 1 (1975).
${ }^{20}$ P. B. Coates, Rev. Sci. Instrum. 63, 2084 (1992).

${ }^{21}$ Y. Li, B. Sztáray, and T. Baer, J. Am. Chem. Soc. 124, 5843 (2002).

${ }^{22}$ R. Y. L. Chim, R. P. Kennedy, R. P. Tuckett, W. Zhou, G. K. Jarvis, D. J. Collins, and P. A. Hatherly, J. Phys. Chem. A 105, 8403 (2001).

${ }^{23}$ Handbook of Physics, edited by W. Benenson, J. W. Harris, H. Stocker, and H. Lutz (American Institute of Physics, Melville, 2006), p. 1102.

${ }^{24}$ Wolfram Research, Inc., Mathematica Edition: Version 4.1, Wolfram Research, Inc., Champaign, Illinois, 2001.

${ }^{25}$ A. Bodi, J. P. Kercher, C. Bond, P. Meteesatien, B. Sztáray, and T. Baer, J. Phys. Chem. A 110, 13425 (2007). 